\title{
An analytical approach to evaluating monotonic functions of fuzzy numbers
}

\author{
Arthur Seibel, Josef Schlattmann \\ Workgroup on System Technologies and Engineering Design Methodology \\ Hamburg University of Technology, 21073 Hamburg, Germany \\ E-mail: arthur.seibel@tuhh.de
}

\begin{abstract}
This paper presents a practical analytical approach to evaluating continuous, monotonic functions of independent fuzzy numbers. The approach is based on a parametric $\alpha$-cut representation of fuzzy numbers and allows for the inclusion of parameter uncertainties into mathematical models.
\end{abstract}

Keywords: Engineering design computations with uncertain parameters, monotonic functions of fuzzy numbers, analytical fuzzy calculus

\section{Introduction}

There is an increasing effort in the scientific community to provide suitable methods for the inclusion of uncertainties into mathematical models. One way to do so is to introduce parametric uncertainty by representing the uncertain model parameters as fuzzy numbers [1] and evaluating the model equations by means of ZADEH's extension principle [2]. The evaluation of this classical formulation of the extension principle, however, turns out to be a highly complex task [3]. Fortunately, BUCKLEY \& QU [4] provide an alternative formulation that operates on $\alpha$-cuts and is applicable to continuous functions of independent fuzzy numbers. Powerful numerical techniques have been developed to implement this alternative formulation [5]. These techniques are particularly suitable for very complex simulation models [6]. In engineering design [7], however, the mathematical equations are usually less complex and hence analytical methods might be more suitable for the inclusion of parameter uncertainties into the computations. For this purpose, a practical analytical approach to evaluating continuous, monotonic functions of independent fuzzy numbers is introduced in this paper, which is based on the alternative formulation of the extension principle.

An outline of this paper is as follows: In Section 2 , we give a definition of fuzzy numbers and present two important types. In Section 3, we briefly recall ZADEH's extension principle and introduce the alternative formulation based on $\alpha$-cuts. In Section 4 , we describe our analytical approach and give three illustrative examples. In Section 5, a practical engineering application is presented. Finally, in Section 6 , some conclusions are drawn.

\section{Fuzzy numbers}

Fuzzy numbers [1] are a special class of fuzzy sets [8], which can be defined as follows:

A normal, convex fuzzy set $\tilde{x}$ over the real line $\mathbb{R}$ is called fuzzy number if there is exactly one $\bar{x} \in \mathbb{R}$ with $\mu_{\tilde{x}}(\bar{x})=1$ and the membership function is at least piecewise continuous. The value $\bar{x}$ is called the modal or peak value of $\tilde{x}$.

Theoretically, an infinite number of possible types of fuzzy numbers can be defined. However, only few of them are important for engineering applications [6]. These typical fuzzy numbers shall be described in the following.

\subsection{Triangular fuzzy numbers}

Due to its very simple, linear membership function, the triangular fuzzy number (TFN) is the most frequently used fuzzy number in engineering. In order to define a TFN with the membership function

$$
\mu_{\tilde{x}}(x)= \begin{cases}1+\frac{x-\bar{x}}{\tau^{\mathrm{L}}}, & \bar{x}-\tau^{\mathrm{L}} \leq x \leq \bar{x}, \\ 1-\frac{x-\bar{x}}{\tau^{\mathrm{R}}}, & \bar{x}<x \leq \bar{x}+\tau^{\mathrm{R}},\end{cases}
$$

we use the parametric notation [6]

$$
\tilde{x}=\operatorname{tfn}\left(\bar{x}, \tau^{\mathrm{L}}, \tau^{\mathrm{R}}\right),
$$

where $\bar{x}$ denotes the modal value, $\tau^{\mathrm{L}}$ the left-hand, and $\tau^{\mathrm{R}}$ the right-hand spread of $\tilde{x}$ (cf. Figure 1 ). If $\tau^{\mathrm{L}}=\tau^{\mathrm{R}}$, the TFN is called symmetric. Its $\alpha$-cuts $x(\alpha)=\left[x^{\mathrm{L}}(\alpha), x^{\mathrm{R}}(\alpha)\right]$ result from the inverse functions of Eqs. (1) with respect to $x$ :

$$
\begin{array}{ll}
x^{\mathrm{L}}(\alpha)=\bar{x}-\tau^{\mathrm{L}}(1-\alpha), & 0<\alpha \leq 1, \\
x^{\mathrm{R}}(\alpha)=\bar{x}+\tau^{\mathrm{R}}(1-\alpha), & 0<\alpha \leq 1 .
\end{array}
$$

\subsection{Gaussian fuzzy numbers}

Another widely-used fuzzy number in engineering is the Gaussian fuzzy number (GFN), which is based on the normal distribution from probability theory. In order to define such a GFN with the membership function

$$
\mu_{\tilde{x}}(x)= \begin{cases}\exp \left[-\frac{1}{2}\left(\frac{x-\bar{x}}{\sigma^{\mathrm{L}}}\right)^{2}\right], & x \leq \bar{x}, \\ \exp \left[-\frac{1}{2}\left(\frac{x-\bar{x}}{\sigma^{\mathrm{R}}}\right)^{2}\right], & x>\bar{x}\end{cases}
$$




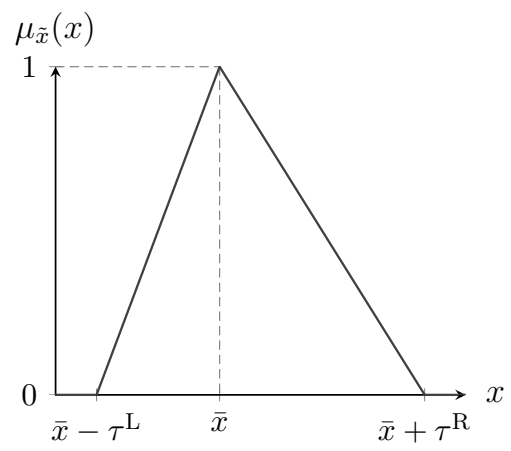

Figure 1: Triangular fuzzy number.

we use the parametric notation [6]

$$
\tilde{x}=\operatorname{gfn}\left(\bar{x}, \sigma^{\mathrm{L}}, \sigma^{\mathrm{R}}\right),
$$

where $\bar{x}$ denotes the modal value, $\sigma^{\mathrm{L}}$ the left-hand, and $\sigma^{\mathrm{R}}$ the right-hand standard deviation of $\tilde{x}$ (cf. Figure 2). If $\sigma^{\mathrm{L}}=\sigma^{\mathrm{R}}$, the GFN is called symmetric. Its $\alpha$-cuts $x(\alpha)=\left[x^{\mathrm{L}}(\alpha), x^{\mathrm{R}}(\alpha)\right]$ result to:

$$
\begin{array}{ll}
x^{\mathrm{L}}(\alpha)=\bar{x}-\sigma^{\mathrm{L}} \sqrt{-2 \ln (\alpha)}, & 0<\alpha \leq 1, \\
x^{\mathrm{R}}(\alpha)=\bar{x}+\sigma^{\mathrm{R}} \sqrt{-2 \ln (\alpha)}, & 0<\alpha \leq 1 .
\end{array}
$$

\section{Extension principle}

ZADEH's extension principle [2] allows to extend any real-valued function to a function of fuzzy numbers. More specifically, let $\tilde{x}_{1}, \ldots, \tilde{x}_{n}$ be $n$ independent or non-interactive fuzzy numbers and let $f: \mathbb{R}^{n} \rightarrow \mathbb{R}$ be a function with $y=f\left(x_{1}, \ldots, x_{n}\right)$. The fuzzy extension $\tilde{y}=f\left(\tilde{x}_{1}, \ldots, \tilde{x}_{n}\right)$ is then defined by

$$
\mu_{\tilde{y}}(y)=\sup _{y=f\left(x_{1}, \ldots, x_{n}\right)} \min \left\{\mu_{\tilde{x}_{1}}\left(x_{1}\right), \ldots, \mu_{\tilde{x}_{n}}\left(x_{n}\right)\right\} .
$$

In case of interdependency between $\tilde{x}_{1}, \ldots, \tilde{x}_{n}$, the minimum operator should be replaced by a suitable triangular norm [9]. In this paper, however, we restrict ourselves to independent fuzzy numbers.

The evaluation of this classical formulation of the extension principle turns out to be a highly complex task [3]. Fortunately, BUCKLEY \& QU [4] provide an alternative formulation that operates on $\alpha$-cuts:

Let $x_{1}(\alpha), \ldots, x_{n}(\alpha)$ denote the $\alpha$-cuts of the $n$ independent fuzzy numbers $\tilde{x}_{1}, \ldots, \tilde{x}_{n}$ and let $f$ be continuous. Then, the $\alpha$-cuts $y(\alpha)=\left[y^{\mathrm{L}}(\alpha), y^{\mathrm{R}}(\alpha)\right]$ of $\tilde{y}$ can be computed from

$$
\begin{aligned}
y^{\mathrm{L}}(\alpha) & =\min \left\{f\left(x_{1}, \ldots, x_{n}\right) \mid\left(x_{1}, \ldots, x_{n}\right) \in \Omega(\alpha)\right\}, \\
y^{\mathrm{R}}(\alpha) & =\max \left\{f\left(x_{1}, \ldots, x_{n}\right) \mid\left(x_{1}, \ldots, x_{n}\right) \in \Omega(\alpha)\right\},
\end{aligned}
$$

where $\Omega(\alpha)=x_{1}(\alpha) \times \cdots \times x_{n}(\alpha)$ represent the $n$ dimensional interval boxes that are spanned by the $\alpha$-cuts $x_{1}(\alpha), \ldots, x_{n}(\alpha)$.

The proposed analytical approach, which is presented in the next section, is based on this alternative formulation of the extension principle.

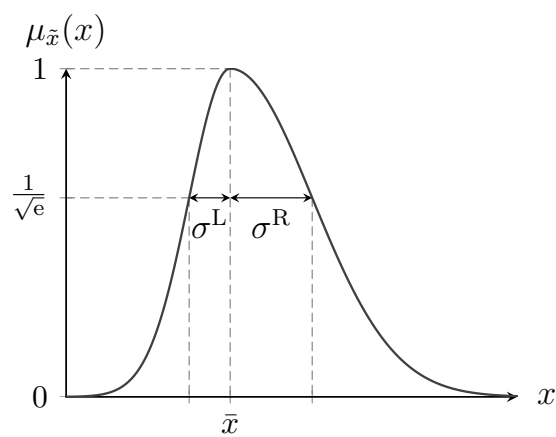

Figure 2: Gaussian fuzzy number.

\section{Analytical approach}

Let the continuous function $f$ be (strictly) monotonic increasing in $x_{i}, i=1, \ldots, k$, and (strictly) monotonic decreasing in $x_{j}, j=1, \ldots, \ell$, in the domain of interest, and let $k+\ell=n$. Then, the minimum values of $f$ inside of every sub-domain $\Omega(\alpha)$ are always found at the left boundaries of $x_{i}(\alpha)$ and the right boundaries of $x_{j}(\alpha)$, and its maximum values at the right boundaries of $x_{i}(\alpha)$ and the left boundaries of $x_{j}(\alpha)$, respectively. In such case, the $\alpha$-cuts $y(\alpha)=$ $\left[y^{\mathrm{L}}(\alpha), y^{\mathrm{R}}(\alpha)\right]$ of $\tilde{y}$ become

$$
\begin{array}{ll}
y^{\mathrm{L}}(\alpha)=f\left(x_{i}^{\mathrm{L}}(\alpha), x_{j}^{\mathrm{R}}(\alpha)\right), & 0<\alpha \leq 1, \\
y^{\mathrm{R}}(\alpha)=f\left(x_{i}^{\mathrm{R}}(\alpha), x_{j}^{\mathrm{L}}(\alpha)\right), & 0<\alpha \leq 1,
\end{array}
$$

with $x_{m}(\alpha)=\left[x_{m}^{\mathrm{L}}(\alpha), x_{m}^{\mathrm{R}}(\alpha)\right], m=1, \ldots, n$. If Eqs. (2) are invertible with respect to $\alpha$, then the membership function of $\tilde{y}$ yields

$$
\mu_{\tilde{y}}(y)= \begin{cases}y^{\mathrm{L}}(\alpha)^{-1}, & y^{\mathrm{L}}(0)<y \leq y^{\mathrm{L}}(1), \\ y^{\mathrm{R}}(\alpha)^{-1}, & y^{\mathrm{R}}(1)<y<y^{\mathrm{R}}(0) .\end{cases}
$$

This reduced part of our approach can be viewed as an analytical version of the short transformation method [10]. Basically, it is equivalent to Lemma 3 from [11] or Corollary 2 from [12].

Example 1. The function $f_{1}: \mathbb{R}_{+}^{2} \rightarrow \mathbb{R}_{+}$with

$$
y_{1}=f_{1}\left(x_{1}, x_{2}\right)=\frac{x_{1}}{x_{1}+x_{2}}
$$

shall be evaluated for the two fuzzy numbers $\tilde{x}_{1}=$ $\operatorname{tfn}(2,2,3)$ and $\tilde{x}_{2}=\operatorname{tfn}(2,2,2)$. Since

$$
\begin{aligned}
& \frac{\partial f_{1}}{\partial x_{1}}=\frac{x_{2}}{\left(x_{1}+x_{2}\right)^{2}}>0 \\
& \frac{\partial f_{1}}{\partial x_{2}}=\frac{-x_{1}}{\left(x_{1}+x_{2}\right)^{2}}<0
\end{aligned}
$$

the function $f_{1}$ is (strictly) monotonic increasing in $x_{1}$ and (strictly) monotonic decreasing in $x_{2}$ in the domain $\operatorname{supp}\left(\tilde{x}_{1}\right) \times \operatorname{supp}\left(\tilde{x}_{2}\right)=(0,5) \times(0,4)$. Hence, the $\alpha$-cuts $y_{1}(\alpha)=\left[y_{1}^{\mathrm{L}}(\alpha), y_{1}^{\mathrm{R}}(\alpha)\right]$ of $\tilde{y}_{1}$ are

$$
y_{1}^{\mathrm{L}}(\alpha)=f_{1}\left(x_{1}^{\mathrm{L}}(\alpha), x_{2}^{\mathrm{R}}(\alpha)\right)=\frac{1}{2} \alpha,
$$




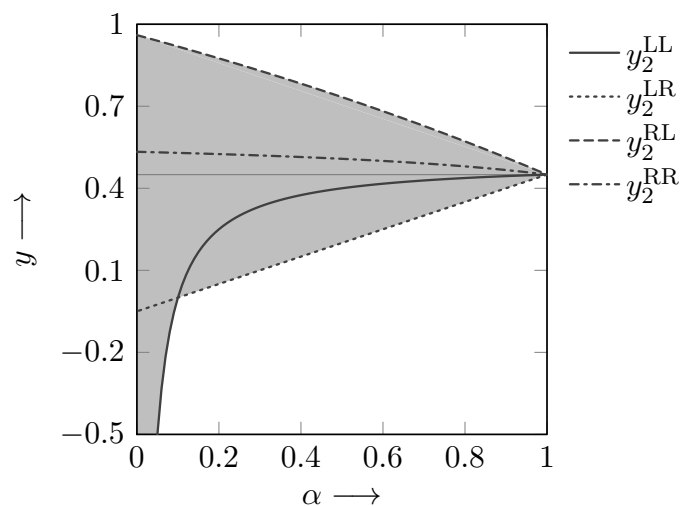

Figure 3: Solution candidates from Example 2.

$$
y_{1}^{\mathrm{R}}(\alpha)=f_{1}\left(x_{1}^{\mathrm{R}}(\alpha), x_{2}^{\mathrm{L}}(\alpha)\right)=\frac{3 \alpha-5}{\alpha-5} .
$$

With $y_{1}^{\mathrm{L}}(0)=0, y_{1}^{\mathrm{L}}(1)=0.5=y_{1}^{\mathrm{R}}(1)$, and $y_{1}^{\mathrm{R}}(0)=1$, the membership function of $\tilde{y}_{1}$ yields

$$
\mu_{\tilde{y}_{1}}(y)= \begin{cases}2 y, & 0<y \leq 0.5 \\ \frac{5(y-1)}{y-3}, & 0.5<y<1 .\end{cases}
$$

However, the above approach is only valid if the function $f$ does not change its monotonicity within the domain of interest. We know from $[13,14]$ that the global extrema of any monotonic function $f$ are always found at the corner points of $\Omega(\alpha)$. Hence, in order to compute the analytical solution, we can always proceed as follows:

1. Evaluate the function $f$ for all the $2^{n}$ permutations of the interval boundaries of $x_{m}(\alpha)$; e.g., if $n=2$, then compute

$$
\begin{aligned}
& y^{\mathrm{LL}}(\alpha)=f\left(x_{1}^{\mathrm{L}}(\alpha), x_{2}^{\mathrm{L}}(\alpha)\right), \\
& y^{\mathrm{LR}}(\alpha)=f\left(x_{1}^{\mathrm{L}}(\alpha), x_{2}^{\mathrm{R}}(\alpha)\right), \\
& y^{\mathrm{RL}}(\alpha)=f\left(x_{1}^{\mathrm{R}}(\alpha), x_{2}^{\mathrm{L}}(\alpha)\right), \\
& y^{\mathrm{RR}}(\alpha)=f\left(x_{1}^{\mathrm{R}}(\alpha), x_{2}^{\mathrm{R}}(\alpha)\right) .
\end{aligned}
$$

2. Plot these solution candidates in the same diagram.

3. The analytical solution then corresponds to the maximum envelope formed by the possible solution candidates.

This general part of our approach can be viewed as an analytical version of the reduced transformation method [15]. Basically, it is equivalent to Lemma 2 from [11] or Corollary 1 from [12].

Example 2. Next, the function $f_{2}: \mathbb{R}_{+}^{2} \rightarrow \mathbb{R}$ with

$$
y_{2}=f_{2}\left(x_{1}, x_{2}\right)=\frac{x_{1}-\frac{1}{5}}{x_{1}+x_{2}}
$$

shall be evaluated for the two fuzzy numbers from Example 1. Since

$$
\frac{\partial f_{2}}{\partial x_{1}}=\frac{x_{2}+\frac{1}{5}}{\left(x_{1}+x_{2}\right)^{2}}>0, \quad 0<x_{2}<4,
$$

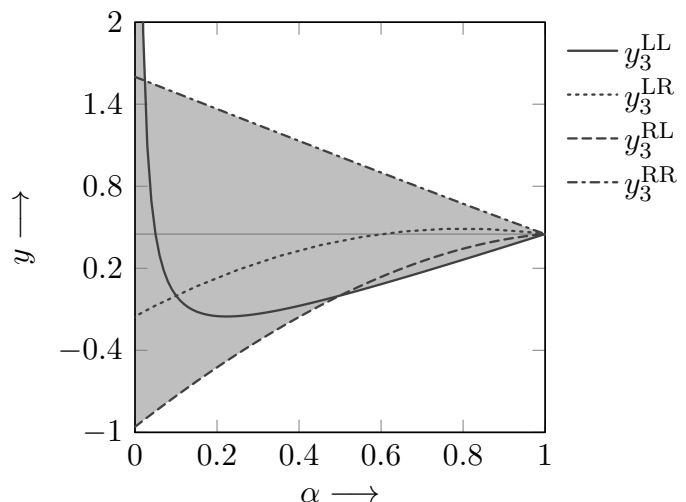

Figure 4: Solution candidates from Example 3.

$$
\frac{\partial f_{2}}{\partial x_{2}}=\frac{\frac{1}{5}-x_{1}}{\left(x_{1}+x_{2}\right)^{2}} \begin{cases}\geq 0, & 0<x_{1} \leq 0.2, \\ <0, & 0.2<x_{1}<5,\end{cases}
$$

the function $f_{2}$ changes its monotonicity within the domain $\operatorname{supp}\left(\tilde{x}_{1}\right) \times \operatorname{supp}\left(\tilde{x}_{2}\right)=(0,5) \times(0,4)$. Hence, the general part of our approach should be applied. The solution candidates for $y_{2}(\alpha)$ are

$$
\begin{aligned}
y_{2}^{\mathrm{LL}}(\alpha)=f_{2}\left(x_{1}^{\mathrm{L}}(\alpha), x_{2}^{\mathrm{L}}(\alpha)\right) & =\frac{10 \alpha-1}{20 \alpha}, \\
y_{2}^{\mathrm{LR}}(\alpha)=f_{2}\left(x_{1}^{\mathrm{L}}(\alpha), x_{2}^{\mathrm{R}}(\alpha)\right) & =\frac{1}{2} \alpha-\frac{1}{20}, \\
y_{2}^{\mathrm{RL}}(\alpha)=f_{2}\left(x_{1}^{\mathrm{R}}(\alpha), x_{2}^{\mathrm{L}}(\alpha)\right) & =\frac{3}{5} \frac{5 \alpha-8}{\alpha-5}, \\
y_{2}^{\mathrm{RR}}(\alpha)=f_{2}\left(x_{1}^{\mathrm{R}}(\alpha), x_{2}^{\mathrm{R}}(\alpha)\right) & =\frac{3}{5} \frac{5 \alpha-8}{5 \alpha-9} .
\end{aligned}
$$

We can see from their plots in Figure 3 that the left branch of the maximum envelope, illustrated by the gray area, is formed by $y_{2}^{\mathrm{LL}}$ for $0<\alpha \leq 0.1$ and by $y_{2}^{\mathrm{LR}}$ for $0.1<\alpha \leq 1$, where the value 0.1 corresponds to their intersection point. Its right branch, on the other hand, is entirely formed by $y_{2}^{\mathrm{RL}}$. Hence, the $\alpha$-cuts $y_{2}(\alpha)=\left[y_{2}^{\mathrm{L}}(\alpha), y_{2}^{\mathrm{R}}(\alpha)\right]$ of $\tilde{y}_{2}$ are

$$
\begin{aligned}
& y_{2}^{\mathrm{L}}(\alpha)= \begin{cases}\frac{10 \alpha-1}{20 \alpha}, & 0<\alpha \leq 0.1, \\
\frac{1}{2} \alpha-\frac{1}{20}, & 0.1<\alpha \leq 1,\end{cases} \\
& y_{2}^{\mathrm{R}}(\alpha)=\frac{3}{5} \frac{5 \alpha-8}{\alpha-5}, \quad 0<\alpha \leq 1 .
\end{aligned}
$$

With $\lim _{\alpha \rightarrow 0} y_{2}^{\mathrm{L}}(\alpha)=-\infty, y_{2}^{\mathrm{L}}(0.1)=0, y_{2}^{\mathrm{L}}(1)=$ $0.45=y_{2}^{\mathrm{R}}(1)$, and $y_{2}^{\mathrm{R}}(0)=0.96$, the membership function of $\tilde{y}_{2}$ yields

$$
\mu_{\tilde{y}_{2}}(y)= \begin{cases}\frac{1}{10} \frac{1}{1-2 y}, & -\infty<y \leq 0, \\ 2 y+\frac{1}{10}, & 0<y \leq 0.45, \\ \frac{1}{5} \frac{25 y-24}{y-3}, & 0.45<y<0.96 .\end{cases}
$$

Example 3. Finally, the function $f_{3}: \mathbb{R}_{+}^{2} \rightarrow \mathbb{R}$ with

$$
y_{3}=f_{3}\left(x_{1}, x_{2}\right)=\frac{\left(x_{1}-\frac{1}{5}\right)\left(x_{2}-1\right)}{x_{1}+x_{2}}
$$


shall be evaluated for the two fuzzy numbers from Example 1. Since

$$
\begin{aligned}
& \frac{\partial f_{3}}{\partial x_{1}}=\frac{\left(x_{2}-1\right)\left(x_{2}+\frac{1}{5}\right)}{\left(x_{1}+x_{2}\right)^{2}} \begin{cases}\leq 0, & 0<x_{2} \leq 1, \\
>0, & 1<x_{2}<4,\end{cases} \\
& \frac{\partial f_{3}}{\partial x_{2}}=\frac{\left(x_{1}-\frac{1}{5}\right)\left(x_{1}+1\right)}{\left(x_{1}+x_{2}\right)^{2}} \begin{cases}\leq 0, & 0<x_{1} \leq 0.2, \\
>0, & 0.2<x_{1}<5,\end{cases}
\end{aligned}
$$

the function $f_{3}$ changes its monotonicity within the domain $\operatorname{supp}\left(\tilde{x}_{1}\right) \times \operatorname{supp}\left(\tilde{x}_{2}\right)=(0,5) \times(0,4)$. Hence, the general part of our approach should be applied. The solution candidates for $y_{3}(\alpha)$ are

$$
\begin{gathered}
y_{3}^{\mathrm{LL}}(\alpha)=f_{3}\left(x_{1}^{\mathrm{L}}(\alpha), x_{2}^{\mathrm{L}}(\alpha)\right)=\frac{(10 \alpha-1)(2 \alpha-1)}{20 \alpha}, \\
y_{3}^{\mathrm{LR}}(\alpha)=f_{3}\left(x_{1}^{\mathrm{L}}(\alpha), x_{2}^{\mathrm{R}}(\alpha)\right)=\frac{(10 \alpha-1)(3-2 \alpha)}{20}, \\
y_{3}^{\mathrm{RL}}(\alpha)=f_{3}\left(x_{1}^{\mathrm{R}}(\alpha), x_{2}^{\mathrm{L}}(\alpha)\right)=\frac{3}{5} \frac{(5 \alpha-8)(2 \alpha-1)}{\alpha-5}, \\
y_{3}^{\mathrm{RR}}(\alpha)=f_{3}\left(x_{1}^{\mathrm{R}}(\alpha), x_{2}^{\mathrm{R}}(\alpha)\right)=\frac{3}{5} \frac{(5 \alpha-8)(3-2 \alpha)}{5 \alpha-9} .
\end{gathered}
$$

We can see from their plots in Figure 4 that the left branch of the maximum envelope is formed by $y_{3}^{\mathrm{RL}}$ for $0<\alpha \leq 0.5$ and by $y_{3}^{\mathrm{LL}}$ for $0.5<\alpha \leq 1$, where the value 0.5 corresponds to the intersection point between $y_{3}^{\mathrm{RL}}$ and $y_{3}^{\mathrm{LL}}$. Its right branch, on the other hand, is formed by $y_{3}^{\mathrm{LL}}$ for $0<\alpha \leq 0.02$ and by $y_{3}^{\mathrm{RR}}$ for $0.02<\alpha \leq 1$, where the value 0.02 corresponds to the intersection point between $y_{3}^{\mathrm{LL}}$ and $y_{3}^{\mathrm{RR}}$. Note that the value 0.02 is only approximate. Hence, the $\alpha$-cuts $y_{3}(\alpha)=\left[y_{3}^{\mathrm{L}}(\alpha), y_{3}^{\mathrm{R}}(\alpha)\right]$ of $\tilde{y}_{3}$ are

$$
\begin{gathered}
y_{3}^{\mathrm{L}}(\alpha)= \begin{cases}\frac{3}{5} \frac{(5 \alpha-8)(2 \alpha-1)}{\alpha-5}, & 0<\alpha \leq 0.5, \\
\frac{(10 \alpha-1)(2 \alpha-1)}{20 \alpha}, & 0.5<\alpha \leq 1,\end{cases} \\
y_{3}^{\mathrm{R}}(\alpha)= \begin{cases}\frac{(10 \alpha-1)(2 \alpha-1)}{20 \alpha}, & 0<\alpha \leq 0.02, \\
\frac{3}{5} \frac{(5 \alpha-8)(3-2 \alpha)}{5 \alpha-9}, & 0.02<\alpha \leq 1 .\end{cases}
\end{gathered}
$$

With $y_{3}^{\mathrm{L}}(0)=-0.96, y_{3}^{\mathrm{L}}(0.5)=0, y_{3}^{\mathrm{L}}(1)=0.45=$ $y_{3}^{\mathrm{R}}(1), y_{3}^{\mathrm{R}}(0.02) \approx 1.57$, and $\lim _{\alpha \rightarrow 0} y_{3}^{\mathrm{R}}(\alpha)=\infty$, the membership function of $\tilde{y}_{3}$ yields

$$
\mu_{\tilde{y}_{3}}(y)= \begin{cases}\frac{21}{20}+\frac{1}{12} y-\frac{1}{60} \sqrt{A}, & -0.96<y \leq 0, \\ \frac{3}{10}+\frac{1}{2} y+\frac{1}{10} \sqrt{B}, & 0<y \leq 0.45, \\ \frac{31}{20}-\frac{5}{12} y-\frac{1}{60} \sqrt{C}, & 0.45<y \leq 1.57, \\ \frac{3}{10}+\frac{1}{2} y-\frac{1}{10} \sqrt{D}, & 1.57<y<\infty,\end{cases}
$$

where

$$
\begin{aligned}
& A=25 y^{2}-2370 y+1089 \\
& B=25 y^{2}+30 y+4 \\
& C=625 y^{2}+750 y+9 \\
& D=25 y^{2}+30 y+4
\end{aligned}
$$

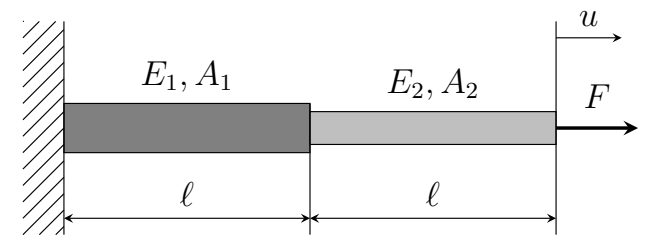

Figure 5: Two-component massless rod.

\section{Engineering application}

In order to illustrate the analytical approach in a more practical context, we consider a rather simple but typical example from engineering mechanics [6] consisting of a two-component massless rod under tensile load as shown in Figure 5. The components of the rod are characterized by the length $\ell$, the elastic moduli $E_{1}$ and $E_{2}$, and the cross sectional areas $A_{1}$ and $A_{2}$. The left component of the rod is clamped to a wall, whereas the right component is subjected to a tensile force $F$. In order to compute the (static) displacement $u$ of the tip of the rod, we can proceed as follows:

Every component of the rod can be viewed as a spring with the stiffness

$$
c_{i}=\frac{E_{i} \cdot A_{i}}{\ell}, \quad i=1,2 .
$$

Since both components are placed in a row, the total stiffness $c_{\text {tot }}$ of the rod is

$$
\frac{1}{c_{\mathrm{tot}}}=\frac{1}{c_{1}}+\frac{1}{c_{2}}
$$

According to Hook's law, the displacement $u$ of the tip of the rod yields

$$
u=\frac{F}{c_{\mathrm{tot}}}=\left(\frac{1}{c_{1}}+\frac{1}{c_{2}}\right) F .
$$

At first, the displacement $u$ shall be computed for crisp parameters $c_{1}$ and $c_{2}$, where the first component is assumed to be made of steel and the second component of aluminum with the following material and geometry values:

$$
\begin{array}{ll}
E_{1}=2.0 \cdot 10^{5} \mathrm{~N} / \mathrm{mm}^{2}, & A_{1}=100 \mathrm{~mm}^{2}, \\
E_{2}=6.9 \cdot 10^{4} \mathrm{~N} / \mathrm{mm}^{2}, & A_{2}=75 \mathrm{~mm}^{2},
\end{array}
$$

and $\ell=500 \mathrm{~mm}$. The external force shall have the absolute value $F=1000 \mathrm{~N}$. With these values, the (crisp) solution for the displacement $u$ yields

$$
\bar{u} \approx 0.1216 \mathrm{~mm} \text {. }
$$

In reality, however, exact stiffness values for both rod components can usually not be provided due to variations in the manufacturing process. In order to include these uncertainties into the computation, the stiffness parameters $c_{1}$ and $c_{2}$ shall be modeled as symmetric GFNs with the modal values

$$
\begin{aligned}
& \bar{c}_{1}=4.0 \cdot 10^{4} \mathrm{~N} / \mathrm{mm}, \\
& \bar{c}_{2}=1.035 \cdot 10^{4} \mathrm{~N} / \mathrm{mm} .
\end{aligned}
$$




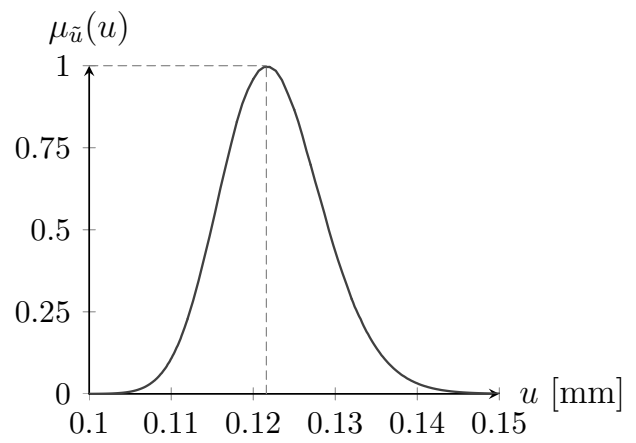

Figure 6: Fuzzy displacement $\tilde{u}$ of the tip of the rod.

The standard deviations of $\tilde{c}_{1}$ and $\tilde{c}_{2}$ are assumed to be $5 \%$ of their modal values. Hence, the parametric representation of the fuzzy stiffness parameters is

$$
\tilde{c}_{i}=\operatorname{gfn}\left(\bar{c}_{i}, 0.05 \bar{c}_{i}, 0.05 \bar{c}_{i}\right), \quad i=1,2 .
$$

In order to compute the $\alpha$-cuts of the fuzzy displacement $\tilde{u}$, we consider again Eq. (3), where we can see that $u$ is (strictly) monotonic decreasing in both $c_{1}$ and $c_{2}$ for positive values. Hence, the $\alpha$-cuts $u(\alpha)=\left[u^{\mathrm{L}}(\alpha), u^{\mathrm{R}}(\alpha)\right]$ of $\tilde{u}$ are

$$
\begin{aligned}
& u^{\mathrm{L}}(\alpha)=u\left(c_{1}^{\mathrm{R}}(\alpha), c_{2}^{\mathrm{R}}(\alpha)\right)=\frac{1007}{414(20+\sqrt{-2 \ln (\alpha)})}, \\
& u^{\mathrm{R}}(\alpha)=u\left(c_{1}^{\mathrm{L}}(\alpha), c_{2}^{\mathrm{L}}(\alpha)\right)=\frac{1007}{414(20-\sqrt{-2 \ln (\alpha)})},
\end{aligned}
$$

and its membership function yields

$$
\mu_{\tilde{u}}(u)=\exp \left[-\frac{1}{a}\left(b-\frac{c}{u}+\frac{d}{u^{2}}\right)\right], \quad u>0,
$$

where

$$
\begin{array}{ll}
a=342792, & b=68558400, \\
c=16675920, & d=1014049 .
\end{array}
$$

The plot of $\mu_{\tilde{u}}(u)$ in the range $0.1 \leq u \leq 0.15$ is illustrated in Figure 6.

\section{Conclusions}

The proposed analytical approach turns out to be a very practical tool for the inclusion of parameter uncertainties into mathematical models. It is valid for continuous, monotonic functions of independent fuzzy numbers, but can also be applied to fuzzy intervals as defined, e.g., in [3].

An analytical solution has the advantage that the degrees of membership of the fuzzy output can be computed for any value within the support, whereas a numerical solution only provides a finite number of values. Furthermore, our approach also allows a symbolic processing of uncertainties.

In further research activities, this approach shall be extended to general, non-monotonic functions of independent fuzzy numbers, where the influence of interdependency shall be investigated as well.

\section{Acknowledgments}

The authors thank the anonymous referees for their valuable comments and the constructive criticism.

\section{References}

[1] D. Dubois and H. Prade. Fuzzy Sets and Systems. Academic Press, New York, 1980.

[2] L. A. Zadeh. The concept of a linguistic variable and its application to approximate reasoning-I. Information Sciences, 8:199-249, 1975.

[3] A. Klimke. Uncertainty modeling using fuzzy arithmetic and sparse grids. $\mathrm{PhD}$ Thesis, University of Stuttgart, 2006.

[4] J. J. Buckley and Y. Qu. On using $\alpha$-cuts to evaluate fuzzy equations. Fuzzy Sets and Systems, 38(3):309-312, 1990.

[5] D. Moens and M. Hanss. Non-probabilistic finite element analysis for parametric uncertainty treatment in applied mechanics: Recent advances. Finite Elements in Analysis and Design, 47(1):4-16, 2011.

[6] M. Hanss. Applied Fuzzy Arithmetic. SpringerVerlag, Berlin, Heidelberg, 2005.

[7] G. Pahl, W. Beitz, J. Feldhusen, and K.-H. Grote. Engineering Design. Springer-Verlag, London, 2007.

[8] L. A. Zadeh. Fuzzy sets. Information and Control, 8:338-353, 1965.

[9] K. Scheerlinck. Metaheuristic versus tailormade approaches to optimization problems in the biosciences. PhD Thesis, Ghent University, 2011.

[10] S. Donders, D. Vandepitte, J. van de Peer, and W. Desmet. Assessment of uncertainty on structural dynamic responses with the short transformation method. Journal of Sound and Vibration, 288(3):523-549, 2005.

[11] H. Q. Yang, H. Yao, and J. D. Jones. Calculating functions of fuzzy numbers. Fuzzy Sets and Systems, 55(3):273-283, 1993.

[12] J. Fortin, D. Dubois, and H. Fargier. Gradual numbers and their application to fuzzy interval analysis. IEEE Transactions on Fuzzy Systems, 16(2):388-402, 2008.

[13] W. M. Dong and F. S. Wong. Fuzzy weighted averages and implementation of the extension principle. Fuzzy Sets and Systems, 21(2):183199, 1987.

[14] W. Dong and H. C. Shah. Vertex method for computing functions of fuzzy variables. Fuzzy Sets and Systems, 24(1):65-78, 1987.

[15] M. Hanss. The transformation method for the simulation and analysis of systems with uncertain parameters. Fuzzy Sets and Systems, 130(3):277-289, 2002. 